\title{
MOLECULAR-GENETIC DIAGNOSIS OF A BULGARIAN FAMILY WITH MULTIPLE ENDOCRINE NEOPLASIA, TYPE IIA
}

\author{
Andrey Kirov ${ }^{1}$, Tihomir Todorov ${ }^{2}$, Lyudmila Angelova ${ }^{3}$, Albena Todorova ${ }^{1}$, Vaniyo Mitev \\ ${ }^{1}$ Department of Medical Chemistry and Biochemistry, Medical University of Sofia \\ ${ }^{2}$ Genetic Medico-Diagnostic Laboratory Genica, Sofia \\ ${ }^{3}$ Department of Pediatric Diseases and Medical Genetics, Medical University of Varna
}

\begin{abstract}
INTRODUCTION: Multiple Endocrine Neoplasia (MEN) is a rare autosomal dominant disease with frequency 1:30 000 and it is classified in 3 subtypes: MEN 2A (Sipple syndrome), familial medullary thyroid carcinoma (FMTC), MEN 2B. The MEN 2A subtype constitutes approximately 70\%-80\% of cases of MEN 2 . The onset age of the MEN 2A is prior to age 35 years. MEN 2A has an increased risk for parathyroid hyperplasia or adenoma. The clinical diagnosis is suspected when at least 2 specific endocrine tumors are detected in one patient or close relatives (MTC, pheochromocytoma, parathyroid adenomas). MTC is suspected in case of increased serum levels of calcitonin. The biochemical phenotype of MTC is revealed between age 5 and 25 (mean age 15 years). The tumor formation in the neck area is between age of 15 and 20. Although there is controversy surrounding the definition of C-Cell Hyperplasia (CCH), its utility to identify or confirm MEN 2 has been essentially replaced by RET (REarranged during Transfection) protooncogene testing. This allows the early surgery interventions, proper genetic counseling and prenatal diagnostics.

MATERIAL AND METHODS: We report on a Caucasian male with complains of general weakness and weight loss (10 kg for 2 month). The patient's mother has been diagnosed at 47 as bilateral pheochromocytomas and struma nodosa and died at age of 51 . In the index patient abdominal ultrasound examination with biopsy of adrenal glands and histological analysis revealed pheochromocytoma on the right side; elevated plasma calcitonin concentration and US scan with biopsy of thyroid tissue detected medular thyroid cancer. The index patient has two elder brothers - it turned out that one of them had been surgically treated for medular thyroid cancer in the past; bilateral pheocromocytomas were detected in the family screening process.
\end{abstract}

Address for correspondence:

Lyudmila Angelova, $M D$, PhD

Laboratory of Medical Genetics,

Department of Paediatrics and Medical Genetics

"St. Marina" Hospital,

Christo Smirnenski 1, Varna, Bulgaria

e-mail: lyudmila_angelova@abv.bg

Received: March 03, 2015

Accepted: June 10, 2015 
Andrey Kirov, Tihomir Todorov, Lyudmila Angelova et al.

RESULTS AND CONCLUSION: MEN 2A diagnosis was suspected and we performed sequencing of RET protooncogene. The genetic analysis of the family affected members revealed c.1902C $>$ G, p.Cys634Trp heterozygous mutation. Unfortunately, two kids in the families of 2 of the brothers have also inherited the mutation. All adult family members were offered genetic counseling. The standard therapy and the prophylactic thyroidectomy include the surgical removal of the thyroid gland, followed by parathyroid gland autotransplantation. In case codon 634 mutation the surgery is recommended before age of 5 years. All affected relatives are going through monitoring for residual disease. The screening protocol includes plasma levels of calcitonin. The stimulation tests are more sensitive than calcitonin measurements alone so they are recommended.

Keywords: RET protooncogene, Multiple Endocrine Neoplasia, case report, cancer, DNA, mutation

\section{INTRODUCTION}

Multiple Endocrine Neoplasia (MEN) is a rare autosomal dominant disease with frequency 1:30 000 and it is classified in 3 subtypes: MEN 2A (Sipple syndrome), familial medullary thyroid carcinoma (FMTC) and MEN 2B (1). The MEN 2A subtype constitutes approximately $70 \%-80 \%$ of cases of MEN 2 (1). The onset age of the MEN 2A is prior to age 35 years. MEN 2A has an increased risk for parathyroid hyperplasia or adenoma. MEN 2A is diagnosed clinically by the occurrence of at least two specific endocrine tumors (medullary carcinoma of the thyroid - MTC, pheochromocytoma or parathyroid adeno$\mathrm{ma} /$ hyperplasia) in a single individual or in close relatives (1). MTC is suspected in case of increased serum levels of calcitonin. It is the principal biochemical marker in MTC; it is used for detection, staging, postoperative management, and prognosis. The increased excretion of catecholamine metabolites during urinalysis is a biochemical marker for pheochromocytoma. The biochemical phenotype of MTC is revealed between age 5 and 25 (mean age 15 years). Parathormone plasma level is elevated in parathyroid hyperplasia or adenoma. Unusual blood levels of calcium and phosphorus may also suggest one of these. They may be suspected also in case of decrease in bone density or kidney stones.

Biochemical testing via measurement of plasma free metanephrines or urinary fractionated metanephrines should be performed in patients suspected of having pheochromocytoma or paraganglioma (PPGL) (2) The tumor formation in the neck area in MTC is between age of 15 and 20 (3).

Affected individuals initially develop primary $\mathrm{C}$-cell hyperplasia $(\mathrm{CCH})$ that progresses to early invasive medullary microcarcinoma, and eventually develop grossly invasive macroscopic MTC. MTC originates in calcitonin-producing cells (C-cells) of the thyroid gland. REarranged during Transfection (RET) gene mutations cause abnormal proliferation and differentiation of the C-cells of the thyroid and the adrenal medulla (4). As CCH is a histological diagnosis, supplementing finding in thyroid pathology, the identification or confirmation of MEN 2 has been essentially based on RET sting (5). The gene is localized on chromosome 10 (10q11.2). The genetic testing could confirm the clinical diagnosis, but it can also detect presymptomatic carriers of RET gene mutations in index patient relatives. This allows proper genetic counseling, prenatal diagnostics and early surgery interventions in familial carrier members.

In the vast majority of cases (95\%), MEN $2 \mathrm{~A}$ is caused by mutations affecting cysteine residues in exon 10 (Codons 609, 611, 618, and 620) and 11 (most commonly, codon 634 - it is detected in $85 \%$ of the patients) of the RET gene (1). The codon 634 mutation was recently reported as the second most common cause in Greece MTC patients (4).

Mutations in the RET codon 634 are causative of cutaneous lichen amyloidosis in some MEN $2 \mathrm{~A}=$ FMTC families (6). The RET gene mutations have high penetrance (85\%); patients develop pheochromocytoma in $50 \%$ and hyperparathyroidism in 20-30\%.

\section{MATERIAL AND METHODS}

We report on a Caucasian male of 35 years with complains of general weakness and weigh loss (10 kg for 2 month) admitted to the Endocrinologic clinics, University hospital St. Marina, Varna. In the index 
patient abdominal ultrasound examination with biopsy of adrenal glands and histological analysis revealed pheochromocytoma on right side; elevated plasma calcitonin concentration and US scan with biopsy of thyroid tissue detected medular thyroid cancer. Familial history and medical documentation showed that the patient's mother has been diagnosed at 47 as bilateral pheochromocytomas and struma nodosa and died at age of 51. The index patient has two elder brothers - 40 and 42 years old (Fig. 1). It turned out that one of them (II 1-4) has been surgically treated for MTC in the past, when hormone substitution therapy was prescribed. Bilateral pheochromocytomas were detected in him during the family screening process. Mild fatigue had started in the oldest brother (II 1-5) and right pheochromocytoma and MTC were diagnosed at the time of the familial investigation. Almost preclinical identification of at-risk siblings led to the diagnosis of MTC and right sided pheocromocytoma in him.

DNA from the index patient and his family (his son of 16 and his kniece of 7 encl.) were extracted through standard salt-precipitation protocol and the molecular-genetic testing of the RET gene was performed in order to confirm the clinically suspected MEN 2A diagnosis. All exons and exon-intron boundaries of the RET gene were amplified through PCR (primers sequence are available upon request), followed by Sanger sequenced using BigDye Terminator v.3.1 (Applied Biosystems, Foster City, CA, USA).

\section{RESULTS AND DISCUSSION}

MEN 2A diagnosis was suspected based on the phenotype data and the autosomal manner of inheritance high risk of recurrence (50\%). A de novo gene mutation is only $5 \%$ or less in index cases with MEN 2A. In our family all five first degree relatives descendants of the ancestor were affected. The clinical phenotype of the familial members and their treatment are described by Hristozov et al, 2013 (7). The disease is associated with RET protooncogene mutation so the index patient, all of his brothers and the two children (patients ID 1-3, 1-4, 1-5, 1-6, 1-7) were referred for genetic testing of the RET gene. All adult family members were offered genetic counseling.

The molecular-genetic testing confirmed the clinical diagnosis of MEN 2A. The genetic anal- ysis of the family affected members and their children showed a heterozygous mutation c.1902C $>\mathrm{G}$, p.Cys634Trp in exon 11 of the RET gene in all of them (Figures 1 and 2).

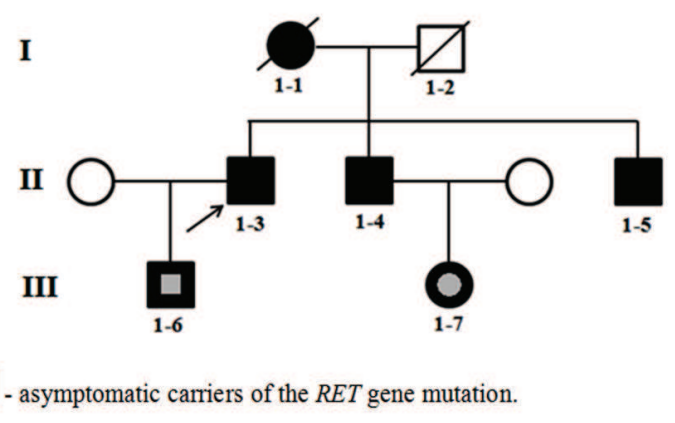

Fig. 1. Family tree of the patient

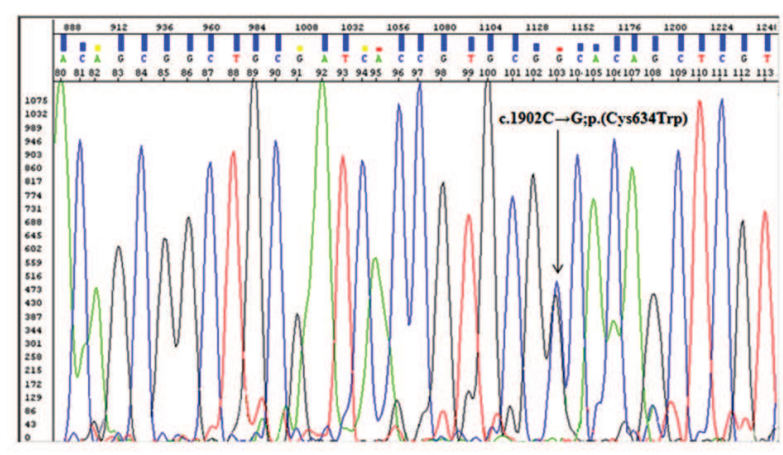

Fig. 2. Result from RET gene molecular genetic testing of the index patient (ID 1-3)

Germline mutations in RET protooncogene have been identified as the underlying cause MEN 2 syndromes and FMTC. This gene encodes for a tyrosine kinase receptor that is only expressed in neural crest-derived tissues (8). Mutation in one of five codons for cysteine in extracellular domain (codons $609,618,620,630$ and 634) in exons 10 and 11 occurs in MEN2A and FMTC. Other mutations less frequently associated with these phenotypes occur in exons 13, 14 and 15 . To date there are no specific mutations responsible only for development of FMTC or MEN 2A phenotype. However, risk for pheochromocytoma in patients with MEN 2A has been associated with any mutation of codon 634 (8).

The molecular-genetic testing showed that all tested relatives (ID 1-3 to 1-7) are carriers of the family mutation. Both children (ID 1-6 and 1-7) are as- 
Andrey Kirov, Tihomir Todorov, Lyudmila Angelova et al.

ymptomatic at the moment and they will go through periodical medical examinations. First, Schuffenecker et al. (9) suggested that all codon 634 mutation carriers are at some risk for hyperparathyroidism and should be screened for this disease. The controversial question for total thyroidectomy is still open, but the fact that $95 \%$ of individuals with MEN 2A (9) develop MTC strongly indicates it. Moreover, bilateral or multifocal areas of MTC and C-cell hyperplasia are usually observed at the time of thyroidectomy in affected individuals undergoing prophylactic thyroidectomy (10). As far as pheochromocytomas usually present after MTC a long term follow-up and extensive screening protocol is needed to determine the correct phenotype and improve patient clinical management.

The standard therapy and the prophylactic thyroidectomy include the surgical removal of the thyroid gland, followed by parathyroid gland autotransplantation (3). In case of codon 634 mutation the surgery is recommended before age of 5 years. All affected relatives are going through monitoring for residual disease. The screening protocol includes plasma levels of calcitonin. The stimulation tests are more sensitive than calcitonin measurements alone so they are recommended.

In case of normal screening results for pheochromocytoma the tests are performed once per year, followed by MRI in case of abnormal results. Abdominal US and CT scan are also recommended as well as screening for parathyroid adenomas once per year $(2,3)$.

Our results allowed classifying the family as MEN 2A, thereby improving clinical management of family members. MEN 2A provides unique model for early prevention of cancer and for stratified roles of mutation-based diagnosis of carriers. Moleculargenetic analysis of MEN 2A provides families with early genetic counseling information (possibilities for prenatal diagnosis included) in high risk dominant cancer predisposing diseases.

\section{REFFERENCE}

1. Moline J, Eng C. Multiple Endocrine Neoplasia Type 2. 1999 Sep 27 [Updated 2013 Jan 10]. In: Pagon RA, Adam MP, Ardinger HH, et al., editors. GeneReviews $^{\oplus}$ [Internet]. Seattle (WA): University of Washington, Seattle; 1993-2015. Available from: http://www.ncbi.nlm.nih.gov/books/NBK1257/

2. Lenders JW, Duh QY, Eisenhofer G, GimenezRoqueplo AP, Grebe SK, Murad MH, et al. Pheochromocytoma and paraganglioma: an endocrine society clinical practice guideline. J Clin Endocrinol Metab. 2014;99(6):1915-42.

3. Friedhelm Raue, Karin Frank-Raue. Genotypephenotype relationship in multiple endocrine neoplasia type 2. Implications for clinical management. Hormones. 2009; 8(1):23-28.

4. Sarika HL, Papathoma A, Garofalaki M et al., Genetic screening of patients with medullary thyroid cancer in a referral center in Greece during the past two decades. Eur J Endocrinol. 2015 Apr;172(4):501-9.

5. American Thyroid Association Guidelines Task Force. Medullary Thyroid Cancer: Management Guidelines of the American Thyroid Association. Thyroid. 2009; 19(6):565-612.

6. Verga U, Fugazzola L, Cambiaghi S, Pritelli C, Alessi E, Cortelazzi D, et al., Frequent association between MEN 2A and cutaneous lichen amyloidosis.Clin Endocrinol. 2003; 59:156-161.

7. Hristozov K, Boyadzhieva M, Radev R, Siderova M, Ivanov K, Krasnaliev I, Kolev N, Nenkov R, Atanasova M. Familia s mnozhestvena endokrinna neoplazia tip 2A. Endokrinologia 2013;18(3):124-130. Bulgarian.

8. Gonzales-Yerba B, M Medrano, A Mantilla, V Palma, C Colin, D Hernandez, J Tapia, B Dawson, M Salcedo. Penetrance of inherited medullary thyroid carcinoma and genotype-phenotype correlation in large MEN type 2A family with C634Y RET mutation. Endocrine Patilogy. 2007;14(1).

9. Schuffenecker I, Virally-Monod M, Brohet R, et al. Risk and penetrance of primary hyperparathyroidism in multiple endocrine neoplasia type $2 \mathrm{~A}$ families with mutations at codon 634 of the RET proto-oncogene. J Clin Endocrinol Metab. 1998 Feb;83(2):487-91.

10. Wiesner G.L. and K. Snow-Bailey. Multiple Emdocrine Neoplasia Type 2. Gene Reviews. Seatle; 2005. 